\title{
A new OIT protocol for severe peanut allergy
}

\author{
AK Kukkonen*, A Pelkonen, M Mäkelä, H Voutilainen, S Mäkinen-Kiljunen, Helsinki University Central Hospital \\ From Food Allergy and Anaphylaxis Meeting (FAAM 2013) \\ Nice, France. 7-9 February 2013
}

\section{Background}

Oal immunotherapy (OIT) may reduce the risk for severe allergic reaction at accidental ingestion of peanut. We aimed at developing a peanut OIT protocol with homebased dose escalations for 6-18 year-old children with moderate to severe allergic reaction in a double-blind placebo-controlled challenge (DBPCFC).

\section{Methods}

Our pirmary outcome was the increase in the amount of ingested peanut protein tolerated in DBPCFC by peanut OIT. Secondarily we investigated safety of OIT. Since September 2011, peanut-sensitized patients with moderate to severe allergic reaction (1) in DBPCFC were included. Bronchial hyperreactivity, exhaled nitric oxide, and food allergy related quality of life $(2,3)$ were evaluated pre and post OIT. Roasted and defatted peanut flour, allergenity of which was assessed using microarray inhibition, was mixed with margarine (no milk or soy) in 3 concentrations at hospital kitchen. A teaspoonful of peanut-margarine mixture weight $1.7 \mathrm{~g}$. From week 20 and on, we used real peanuts. The first dose of $0.1 \mathrm{mg}$ peanut protein was taken at hospital and the same dose continued daily at home. Dose escalations occurred at home every 1 to 2 weeks. The patient returned to the hospital 7 times for dose escalation. Antihistamine was taken daily until 2 weeks from reaching the maintenance dose of 4 peanuts. As safety procedures, exercise was forbidden one hour post each dose and the day of escalation, and the patients carried adrenalin auto-injectors.

\section{Results}

In November 2012, 18 patients mean age $9(6-16)$ y have entered the OIT. At DBPCFC, the median dose of peanut protein causing moderate to severe allergic reaction was $55 \mathrm{mg}$ ( $1 / 4$ peanut), range 5 to $255 \mathrm{mg}, 10 / 18$ had received 1 to 3 doses of adrenalin as treatment for anaphylactic reaction at challenge. The median (range) serum peanut specific-IgE was $89(1.8-817) \mathrm{kU} / \mathrm{L}$ and peanut skin prick test was $10(6-16)$. Fourteen children had asthma. Two patients discontinued the study; one was non-compliant and one had continuous nausea. One patient has received adrenalin after dose escalation at home; 2 patients have taken antihistamines once due to urticaria. Mild oro-pharyngeal itching requiring no medication was common.

\section{Conclusion}

Based on our preliminary results it seems possible to develop a peanut OIT protocol with home-based dose escalations also in anaphylactic patients.

\section{Disclosure of interest}

None declared.

Published: 25 July 2013

\section{References}

1. Hourihane, et al: Clin Exp Allergy 2005, 35:1227-33.

2. Dunn Galvin A, et al: Clin Exp Allergy 2008, 38:977-86.

. Flokstra-de Blok, et al: J Allergy Clin Immunol 2008, 122:139-44.

doi:10.1186/2045-7022-3-S3-P6

Cite this article as: Kukkonen et al:: A new OIT protocol for severe peanut allergy. Clinical and Translational Allergy 2013 3(Suppl 3):P6. 